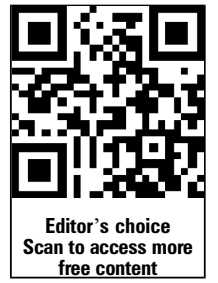

${ }^{1}$ Centre for Physiotherapy Research, School of Physiotherapy, University of Otago, Dunedin, New Zealand ${ }^{2}$ Florey Neurosciences Institutes and the Centre for Health, Exercise and Sports Medicine, University of Melbourne and the Australian Centre for Research in Sports Injury Prevention, Monash University, Melbourne, Australia

\section{Correspondence to} Professor S John Sullivan, Centre for Physiotherapy Research, School of Physiotherapy, University of Otago, PO Box 56, Dunedin 9054, New Zealand; sjohn.sullivan@otago.ac.nz

Received 4 October 2012 Revised 22 December 2012 Accepted 16 January 2013 Published Online First 27 February 2013
To cite: Williams $D$, Sullivan SJ, Schneiders AG, et al. Br J Sports Med 2014;48:107-111.

\title{
Big hits on the small screen: an evaluation of concussion-related videos on YouTube
}

\author{
David Williams, ${ }^{1}$ S John Sullivan, ${ }^{1}$ Anthony G Schneiders, ${ }^{1}$ Osman Hassan Ahmed, ${ }^{1}$ \\ Hopin Lee, ${ }^{1}$ Arun Prasad Balasundaram, ${ }^{1}$ Paul R McCrory ${ }^{2}$
}

\begin{abstract}
Background YouTube is one of the largest social networking websites, allowing users to upload and view video content that provides entertainment and conveys many messages, including those related to health conditions, such as concussion. However, little is known about the content of videos relating to concussion. Objective To identify and classify the content of concussion-related videos available on YouTube.

Study design An observational study using content analysis.

Methods YouTube's video database was systematically searched using 10 search terms selected from MeSH and Google Adwords. The 100 videos with the largest view counts were chosen from the identified videos. These videos and their accompanying text were analysed for purpose, source and description of content by a panel of assessors who classified them into data-driven thematic categories.
\end{abstract}

Results 434 videos met the inclusion criteria and the 100 videos with the largest view counts were chosen. The most common categories of the videos were the depiction of a sporting injury (37\%) and news reports (25\%). News and media organisations were the predominant source (51\%) of concussion-related videos on YouTube, with very few being uploaded by professional or academic organisations. The median number of views per video was 26191.

Conclusions Although a wide range of concussion-related videos were identified, there is a need for healthcare and educational organisations to explore YouTube as a medium for the dissemination of qualitycontrolled information on sports concussion.

\section{INTRODUCTION}

Sport concussions are often poorly understood by those who sustain them, and frequently go unrecognised and unreported. ${ }^{1-4}$ While considerable effort has gone into the generation of best practice information regarding medical concussion management, ${ }^{5} 6$ the challenge remains on how to transfer this information to potential users. The International Concussion in Sport (CIS) Group identified this process of knowledge transfer as a crucial step in the successful and safe return to sport for athletes who have sustained a sports concussion, ${ }^{6}$ and websites and internet-based educational videos have been cited as valuable tools in the transmission of best-practice concussion information. ${ }^{7}$

Endorsement of the use of web-based applications is unsurprising as the internet has seen a global population penetration rise from $23.5 \%$ in 2008 to $32.7 \%$ in 2011, with an estimated 2.3 billion people now enjoying access. ${ }^{8}$ In North America alone, $74 \%$ of adults use the internet daily, ${ }^{9}$ and $80 \%$ of all users have searched for health-related information. ${ }^{10}$

The phenomenon of eHealth (electronic health) is steadily becoming acknowledged as an acceptable and legitimate way of obtaining information about one's health. ${ }^{11}$ However, many studies have highlighted that there is little (if any) regulation of information online, and thus the public has no way of knowing if it is accurate, misleading or commercially targeted. ${ }^{12-14}$

Where the internet was once viewed as an information archive, the development of web 2.0 technologies, as well as the emergence of social networking sites (SNSs) such as Facebook, Twitter and YouTube, has provided users with interactive website arenas that encourage collaboration and the sharing of information. ${ }^{15} 16$ Users are increasingly utilising SNSs as platforms for sourcing and propagating healthcare information, which has given rise to the growth of 'Medicine 2.0'. ${ }^{17} \mathrm{~A}$ review of concussion-related posts on the SNSs Twitter $^{18}$ and Facebook ${ }^{19}$ has shown that users are actively discussing, seeking and sharing knowledge about concussion. SNSs can therefore be seen as a crucial link in the knowledge transfer of concussion-related information within the wider general public community.

YouTube.com is currently the third most accessed website globally after Facebook and Google. ${ }^{20}$ It is the most popular video-sharing website on the internet with 4 billion videos viewed daily ${ }^{21}$ and approximately $60 \mathrm{~h}$ of videos uploaded each minute. ${ }^{22}$ In addition to sharing videos, YouTube is also a popular social network which has its own participatory communities. ${ }^{21-23}$ These communities stimulate social interactions and allow users to communicate with each other on a wide range of topics. The combination of the widespread popularity of YouTube allied to its ability to permit communication between users' highlights its potential role as a powerful and effective tool in the transfer of concussion-related knowledge.

Examination of YouTube video content in the field of healthcare has been reported on topics as diverse as vaccinations, ${ }^{24}$ smoking cessation ${ }^{25}$ and obesity management. ${ }^{10}$ To date, there have been no formal analyses of concussion-related information on YouTube. The aim of this paper was to identify and describe the content and origins of concussionrelated videos on YouTube, in order to provide insight into the nature of concussion videos which the general public are watching. This may indicate whether YouTube is an appropriate and viable medium for the dissemination of concussion-related information. 


\section{METHOD}

When an individual (the uploader) posts a video to YouTube, there is a range of accompanying information associated with the video which assists in locating and describing the video. These include: the title of the video, tags (the equivalent of a keyword) and text which can be used to describe aspects of the video. In addition, viewers can post information (comments) on the video. Collectively, these provide a rich source of information for retrieval and analytical purposes.

\section{Search strategy}

To obtain a representative sample of the range of videos available to the public and healthcare professionals via YouTube, we chose a novel dual strategy to identify appropriate search terms. In order to understand how the public would most likely explore YouTube, we used the Google keyword search tool Google AdWords. ${ }^{26}$ This product, which was developed for advertising purposes, ranks free text search terms based on the number of times the public have used this search term. From the original keyword 'concussion', the terms 'signs of concussion' and 'concussion symptoms' were chosen, and from the keyword 'sports concussion', the terms 'concussion football' and 'concussion treatment' were selected. PubMed's MeSH terms database was also queried in order to obtain search terms relating to concussion. The keyword terms 'athletic injuries', 'neurological manifestations' and 'brain concussion' were chosen and combined with Boolean operators to maximise the permutations. A description of the search strategies is presented in figure 1.

\section{Video selection strategy}

The site 'YouTube.com' was searched on 12 December 2011. As the primary goal was to identify the videos that people are

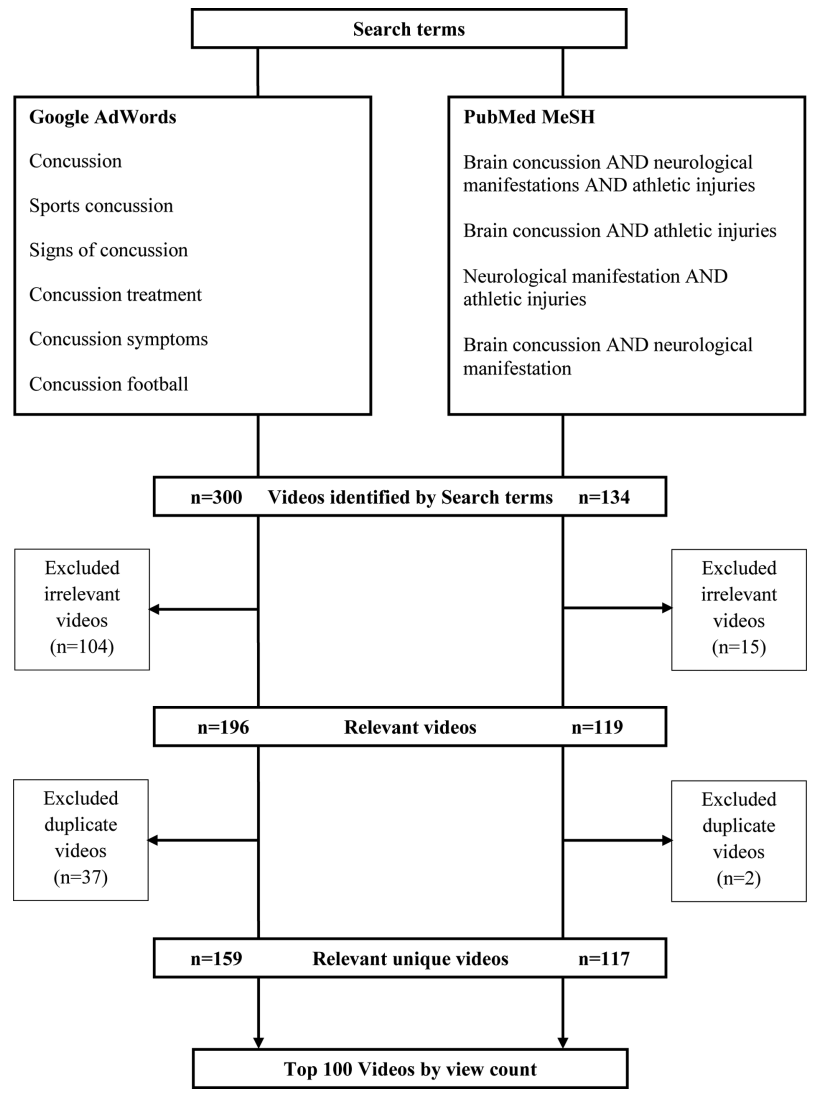

Figure 1 Search strategy flow diagram. watching the most, the YouTube advanced search options of 'sort by view count' and 'video only' were used. The videos were included if they had concussion content, depicted a concussion, discussed a concussion (eg, suffered in a sports situation) or attempted to provide information about concussion. Videos were excluded if they were clearly irrelevant based on the title (eg, music or video games), did not contain concussion content, promoted a particular product without substantive information about a concussion, were not in English or if the content was inappropriate or offensive. In the case of videos containing identical or very similar content, the most viewed video was retained. A list of the top 100 videos was populated, based on view count, and this served as the basis for the subsequent analysis.

\section{Data analysis}

In order to provide a profile of the videos, a range of information was extracted from each video including: the title, uploader's name, YouTube category, first five tags, description excerpt, length, number of views, months since uploaded and the number of comments. These were analysed using descriptive statistics (medians, 25th and 75th percentiles, ranges, frequency counts and percentages). Coding schemes were developed to classify the overall theme of the video, the source (person or organisation which uploaded the video) and the primary purpose of the description which accompanied each video. Classification categories were developed for each of these three objectives based on a preliminary analysis of a wide sample of randomly selected videos which were not part of the analysis dataset although originating from similar SNS studies. ${ }^{9}{ }^{18}$ Categories were refined for the theme, source and description content by the research team, formally defined and inclusion criteria were developed and documented to guide the coding process. A preliminary coding and training trial was conducted using 10 purposefully selected videos and the coding strategy further refined. The definitions of the coding categories are presented in tables 1-3.

Three members of the research team (DW, HL and $\mathrm{AB}$ ) independently coded the 100 selected videos. Each assessor independently viewed and scored the videos in a random order to reduce any systematic coding bias. Assessors assigned a primary code for the theme, source and description. The initial coding data were tabulated and analysed for consistency between assessors by documenting the raw percentage agreement and by

Table 1 Definition, frequency and examples of video themes $(\mathrm{n}=98)$

\begin{tabular}{lll}
\hline Themes & Definition of themes & Number (\%) \\
\hline $\begin{array}{l}\text { Recreational/ } \\
\text { sporting injury }\end{array}$ & $\begin{array}{l}\text { Portrays an injury obtained in a sporting/ } \\
\text { leisure activity } \\
\text { Vews }\end{array}$ & $36(37)$ \\
Educational & $\begin{array}{l}\text { A video from a news/media source } \\
\text { management or attempts to disseminate }\end{array}$ & $24(24)$ \\
& $\begin{array}{l}\text { knowledge of concussion } \\
\text { An injury that occurs during an event } \\
\text { which is posted for entertainment } \\
\text { purposes }\end{array}$ & $11(11)$ \\
Entertainment & $\begin{array}{l}\text { Draws viewer's attention to a product or } \\
\text { service } \\
\text { Video which shares a personal story, }\end{array}$ & $9(9)$ \\
commercial & $\begin{array}{l}\text { situation or experience } \\
\text { Does not fit any other category }\end{array}$ & $2(5)$ \\
\hline $\begin{array}{l}\text { Personal } \\
\text { Onformation }\end{array}$ &
\end{tabular}


Table 2 Definition, frequency and examples of video sources $(\mathrm{n}=98)$

\begin{tabular}{|c|c|c|}
\hline Source & Definition of source & Number (\%) \\
\hline News/media sources & $\begin{array}{l}\text { Unedited video footage of sporting } \\
\text { injury. Recognised news source }\end{array}$ & $50(51)$ \\
\hline $\begin{array}{l}\text { Consumer generated } \\
\text { content }\end{array}$ & Created by a layperson & $29(30)$ \\
\hline $\begin{array}{l}\text { For-profit } \\
\text { organisations }\end{array}$ & Video of commercial origin & $13(13)$ \\
\hline $\begin{array}{l}\text { Advocacy groups/ } \\
\text { non-profit }\end{array}$ & $\begin{array}{l}\text { Link to website which provides free } \\
\text { information-free to join }\end{array}$ & $2(2)$ \\
\hline $\begin{array}{l}\text { Government } \\
\text { agencies }\end{array}$ & $\begin{array}{l}\text { Produced by government agencies (ie, } \\
\text { websites with a .gov domain) }\end{array}$ & $2(2)$ \\
\hline $\begin{array}{l}\text { Medical centres/ } \\
\text { hospitals }\end{array}$ & $\begin{array}{l}\text { Reference to or uploaded by medical } \\
\text { centres/hospitals/departments }\end{array}$ & $1(1)$ \\
\hline $\begin{array}{l}\text { Professional } \\
\text { associations }\end{array}$ & Sporting associations & $1(1)$ \\
\hline
\end{tabular}

calculating the $\kappa(\kappa)$ values for theme $(66.3 \%$ overall agreement; $\kappa=0.60)$, source $(76.9 \%$ overall agreement; $\kappa=0.74)$ and description $(71.1 \%$ overall agreement; $\kappa=0.65)$. The interpretation of the $\kappa$ values followed the criteria proposed by Landis and Koch, ${ }^{27}$ which suggest $\kappa$ values in excess of 0.8 as representing 'excellent' agreement; values between 0.6 and 0.8 as being 'substantial', values between 0.4 and 0.6 as 'moderate' and values less than 0.4 as being 'poor'. For the 10 videos which did not achieve at least a $2 / 3$ majority, two additional members of the research team (JS and AS) joined the original assessors and viewed the videos to determine a final classification. This pragmatic approach to the classification of videos was chosen to provide a robust analysis, in keeping with the challenge of the coding process. The resulting data from this systematic coding were tabulated as frequencies and percentages for descriptive purposes.

\section{RESULTS}

\section{Content analysis}

A total of 434 videos were identified by the search strategy (figure 1). Of these, 119 videos did not meet the inclusion criteria, while a further 39 videos were classed as duplicates of a more popular video and were not included for analysis. From the remaining 276 videos, a 'top 100' list was populated based

Table 3 Definition, frequency and example of description code

\begin{tabular}{|c|c|c|}
\hline Descriptor & Definition of descriptor & Number (\%) \\
\hline $\begin{array}{l}\text { Description of } \\
\text { video content }\end{array}$ & $\begin{array}{l}\text { Descriptive or providing context to video } \\
\text { content or events portrayed }\end{array}$ & $76(78)$ \\
\hline Advertising & $\begin{array}{l}\text { Providing endorsement or information } \\
\text { about a particular product or link to } \\
\text { another non-academic website }\end{array}$ & $8(8)$ \\
\hline $\begin{array}{l}\text { Inferred } \\
\text { management }\end{array}$ & $\begin{array}{l}\text { Description which provides direction for } \\
\text { management for concussion or attempts to } \\
\text { disseminate knowledge about concussion }\end{array}$ & $5(5)$ \\
\hline News & $\begin{array}{l}\text { Text which is a report of news, about } \\
\text { recent or important events. }\end{array}$ & $5(5)$ \\
\hline Other & Does not fit any other category & $3(3)$ \\
\hline $\begin{array}{l}\text { Reference to expert } \\
\text { opinion }\end{array}$ & $\begin{array}{l}\text { Text which notes/mentions an expert in } \\
\text { their respective field or academic article/ } \\
\text { website }\end{array}$ & $1(1)$ \\
\hline
\end{tabular}

on the video's overall view count. This number was pragmatically chosen to provide a sample of approximately $36 \%$ of the total number of videos identified by the search and is in keeping with the sampling percentage used in a previous study of a similar nature. ${ }^{18}$ Of these, 70 came from the Google AdWords keyword search, 12 from the using MeSH terms, while 18 featured in both search strategy results. During the course of the analysis, two videos were removed from YouTube by the uploaders and thus became unavailable for complete analysis. No data from these were included in the analysis, resulting in 98 videos being analysed.

The identified themes which represented the overall purpose of the video are presented in table 1 . The most prevalent theme for the coded videos was that of recreational/sporting injury $(35 / 98)$, which portrayed an individual receiving an injury while participating in sport or recreational activities. This was followed by news (24/98), educational, entertainment, commercial and personal information.

Of the 98 videos analysed, the most common source (50/98) of concussion-related videos was from the news/media organisations/outlets (table 2). Consumer (private) sources accounted for $29(29.59 \%)$ of the videos, while government agencies and advocacy groups were not highly represented.

Table 3 presents categorisation of the text descriptors accompanying each video. Invariably, these were used to describe the video content (76/98), with other categories, such as providing inferred management and referencing an expert, contributing less frequently.

\section{Description of the videos}

The identity of the uploader was often difficult to ascertain. A little over $70 \%$ of the videos were uploaded by individuals from their personal account, or their identity was unable to be determined from the information provided. Sports channels accounted for $11 \%$, as did commercial interests, and health/ medical centres and professional sports bodies featured in $6 \%$ and $1 \%$ of the total, respectively.

The most common categories, as determined by the uploader, were sports $(n=67)$, education $(n=67)$, how to and style $(n=6)$ and news and politics $(n=5)$. The median number of tags assigned to videos was 12 (range 1-60). The most common types of tag referenced the name of an individual athlete or expert, a sporting code, mentioned a head injury and used colloquial terms for a concussion/head injury (table 4).

Although all members of the YouTube community are permitted to upload videos of $15 \mathrm{~min}$ duration or less, videos that are of greater duration can be uploaded on request and by meeting criteria set by the site administrators. As seen in table 5, some videos have remained available for viewing for more than 5 years.

Table 4 Examples of tags associated with the videos

\begin{tabular}{lll}
\hline Tag* & Example of tag & Number (\%) \\
\hline Individual athlete or expert & $\begin{array}{l}\text { Sidney Crosby, Adam James, Max } \\
\text { Pacioretty, Chris Nowinski }\end{array}$ & $90(20)$ \\
A sporting code & $\begin{array}{l}\text { Boxing, Football, Hockey, Rugby } \\
\text { Head trauma, concussion, mild }\end{array}$ & $52(11)$ \\
$\begin{array}{l}\text { Mentioning of a head } \\
\text { injury }\end{array}$ & $\begin{array}{l}\text { traumatic brain injury } \\
\text { Colloquial terms of a } \\
\text { concussion/head injury }\end{array}$ & Knocked out, KO, Hit, Beating \\
\hline
\end{tabular}

*Each video can have more than one tag 
Table 5 Descriptive characteristics of the videos

\begin{tabular}{lllll}
\hline & & & \multicolumn{2}{l}{ IQR (percentiles) } \\
\cline { 3 - 5 } & Median & Range & 25th & 75th \\
\hline Video length & $1 \mathrm{~m} \mathrm{58 \textrm {s }}$ & 14 s to $29 \mathrm{~m} \mathrm{10 \textrm {s }}$ & $1 \mathrm{~m} \mathrm{1 \textrm {s }}$ & $3 \mathrm{~m} \mathrm{48} \mathrm{s}$ \\
Number of views & 26191 & 4629 to 2461849 & 8648 & 244312 \\
Time since posting & 23 months & $1-69$ months & 12 & 40 \\
& 72 & $0-5040$ & 15 & 440 \\
\hline
\end{tabular}

\section{DISCUSSION}

YouTube is a dedicated SNS and has quickly become the medium of choice for the sharing of videos. ${ }^{20} 21$ The simplicity and power of the medium allows both individuals and large organisations to share videos and to communicate their ideas and products. This exploratory study provides an initial insight into how YouTube videos portray a concussion and transmit concussion-related information.

Videos portraying a recreational/sporting injury were the most common theme identified (37\%). This is not entirely surprising, considering that the videos predominately portrayed a professional athlete sustaining an injury. These sporting injuries are ones that users would specifically search for and view, possibly due to the voyeuristic nature of SNS users. ${ }^{28}$ This might explain the large number of comments (5040) recorded for a video, which showed an injury to a high-profile player in a major national basketball competition. A video of a similar nature generated the most views (2 461849 ), where a popular comedian with a large number of SNS subscribers used a video clip of a concussion to a Thai boxer. Furthermore, videos like these are highly likely to be sent as a link to friends and colleagues, and posted on SNSs such as Facebook and Twitter, and the URLs included in blogs, homepages, etc, thus propagating the dissemination of the video. A further 24\% of the videos were news items, demonstrating that YouTube is being used to disseminate concussion-related news stories and supporting the notion that the internet is utilised as part of a multiplatform approach to the gathering and distribution of news information. ${ }^{29}$ This continues from previous research involving the SNS Twitter which showed that people commonly shared concussion-related news stories. ${ }^{18}$ A smaller number of the videos were categorised as educational (11\%) and provided information on the management of a concussion or the dissemination of knowledge about a concussion, such as signs and symptoms, treatment and prevention.

The largest (51\%) source of videos was news or media organisations, indicating their powerful role, intended or otherwise, in the dissemination of concussion-related news. Of note is that consumers generated the second highest $(30 \%)$ number of videos. This is a similar finding to that documented in YouTube research about human papillomavirus (HPV) vaccination. ${ }^{9}$ It is important to note that consumers are producing their own content, independent of media corporations. Consumergenerated content may stimulate discussion and coverage of concussion, that is, independent of new sources. However, consumer-generated content may or may not be from a trustworthy source or be accurate in content. Furthermore, the site (consumer or professionally generated) from which information is obtained has been shown to have a differential outcome. ${ }^{30}$

The video's descriptive text provided a challenge to code as it often contained multiple themes. The majority of the descriptions $(78 \%)$ were used to describe or provide context to the video content. This is hardly surprising as the description is one of the main pieces of information that the YouTube search engine examines for keywords relating to a user's search. Therefore, one of the descriptions' main purposes is to ensure that an uploader's video stands out from other videos and is captured by the search engine. YouTube uploaders are advised to use descriptive language in the video, be accurate and interesting and fill it with as many relevant keywords as possible to distinguish it. ${ }^{31}$ This is often necessary as people performing searches will not know the particular video's title but will enter keywords that relate to a video which could be supplied in the description. Other uses of the description were to advertise a product or service shown in the video $(8 \%)$ or to infer aspects of the management of a concussion (5\%). However, these less prominent codes were also often present in the text but were frequently considered secondary to the descriptive code.

An interesting finding was the way different uploaders utilised the tagging system. A common theme throughout the tags in our dataset was the naming of professional sport's personages $(19.7 \%)$, reinforcing the finding that people are actively uploading and/or searching for videos depicting an athlete. Furthermore, $11 \%$ of tags were of a sporting code such as American football, association football, boxing, etc. In YouTube, a tag is a keyword (120 character limit) which is assigned to a video by the uploader to allow the video to be located by YouTube's search engine. ${ }^{32} 33$ Given their important ability to distinguish a video from others, it would be assumed that uploaders would fully utilise the tags to ensure their video's success. However, it was apparent that some users successfully utilised the tagging system and some tags were underutilised. To fully exploit the potential of the tags, they should be keywords and topics that relate to the title and content of the video. Multiple synonyms of keywords are particularly useful as not all users search using the analogue of a keyword (eg, mild traumatic brain injury, traumatic brain injury and brain damage).

Although YouTube can be seen as a potentially useful medium to disseminate concussion-related knowledge and increase awareness, the user must be aware that the information uploaded is not regulated and the quality of the content is thus questionable. Only 4 of the 11 videos classified as educational (table 1) were from recognised academic sources. Although a formal analysis of the concussion-related content was not performed, it was noted that some information presented did not strictly adhere to that contained in the CIS consensus statement ('when you receive a significant blow to the head') or use appropriate language ('a blow to the noggin'). ${ }^{6}$ Despite these concerns about the quality of information on YouTube, it still retains significant potential to aid in the transfer of concussion-related knowledge.

Unlike previous studies which have used video analysis to understand the mechanisms of sports injuries, ${ }^{34} 35$ this is an initial exploratory study, and future research is needed to better understand this powerful medium and its evolving role as a vehicle in the dissemination of sports medicine information and in the education of the general public. Future studies may be directed to refining the coding categories and addressing more specific issues such as the quality and accuracy of the video content. The scope of YouTube in the dissemination of information has recently been illustrated by the first-hand account of how a health-related video became viral. ${ }^{36}$

This study was not without its limitations, including the small sample size of videos analysed and their manner of selection. Videos were only chosen using the criterion of number of views, and in future studies, videos could be sorted by other 
strategies, such as by relevance. Furthermore, the coding of information is a subjective process, and despite the systematic approach used to obtain consensus, there is the possibility that another group of assessors may have coded the videos differently. Likewise, further refinement of the coding process may have resulted in a stronger consensus between raters. The strengths of the study include the robust and diverse search strategy employed to identify the videos for analysis and the systematic approach to the coding of information.

\section{What are the new findings?}

- Provides an initial insight into the potential of YouTube in disseminating concussion-related information.

- Sensitises sports medicine professionals to the potential of YouTube in delivering health messages.

Acknowledgements The authors acknowledge the funding support from the University of Otago Medical Research Foundation awarded to David Williams in the form of a summer student scholarship.

Contributors All authors contributed to the development of the research, analysis and interpretation of the data, the drafting of the manuscript and approved the final version.

Funding University of Otago Medical Research Foundation summer student scholarship.

Competing interests PM currently receives financial research support from the National Health and Medical Research Council, the University of Melbourne, the Victorian Department of Planning and Community Development, Sport and Recreation Division and the Eastern Health Network. Previous competitive grant funding includes the Australian Research Council, International Rugby Board, the University of Melbourne, the University of Otago (NZ), National Hockey League (US), VicHealth, Australian Football League Research Foundation, Royal Australasian College of Surgeons, and the Australian Sports Commission. He has received travel funding from the Medical Commission of the International Olympic Committee (IOC), the International Football Federation (FIFA), the American Academy of Neurology and the Jockey Club (UK). He receives book royalties from McGraw-Hill and, from 2001 to 2008, was employed by the British Medical Journal Publishing Group. He has conducted clinical drug trials on antimigraine (Glaxo-Welcome; Janssen-Cliag; Novartis; Parke-Davis; Schering) and anti-spasticity drugs (Ipsen) through the Eastern Health Clinical Trials Unit in Melbourne. This drug trial work has not involved any financial payment to Dr McCrory directly. He received consultancy fees in 2010 from Axon Sports (US) for the development of educational material (which was not renewed) and has received support since 2005 from CogState Inc for research costs and the development of educational material. He is a cofounder and shareholder in two biomedical companies (involved in eHealth and Raptor Compression Garment Technologies) but does not hold any individual shares in any company related to social media, concussion or brain injury assessment or technology.

Provenance and peer review Not commissioned; externally peer reviewed.

\section{REFERENCES}

1 McCrea M, Hammeke T, Olsen G, et al. Unreported concussion in high school football players: implications for prevention. Clin J Sport Med 2004;14:13-17.

2 Sullivan SJ, Bourne L, Choie $S$, et al. Understanding of sport concussion by the parents of young rugby players: A pilot study. Clin J Sport Med 2009;19:228-30.

3 Sye G, Sullivan SJ, McCrory P. High school rugby players' understanding of concussion and return to play guidelines. Br J Sports Med 2006;40:1003-4.

4 Williamson IJS, Goodman D. Converging evidence for the under-reporting of concussions in youth ice hockey. Br J Sports Med 2006;40:128-32.
5 McCrory $\mathrm{P}$, Johnston $\mathrm{K}$, Meeuwisse $\mathrm{W}$, et al. Summary and agreement statement of the 2nd International Conference on Concussion in Sport, Prague 2004. Br J Sports Med 2005;39(Suppl 1):78-86.

6 McCrory P, Meeuwisse W, Johnston K, et al. Consensus statement on concussion in sport: the 3rd International Conference on Concussion in Sport held in Zurich November 2008. Br J Sports Med 2009;43(Suppl 1):76-84.

7 Provvidenza $C$, Johnston KM. Knowledge transfer principles as applied to sport concussion education. Br J Sports Med 2009;43(Suppl 1):68-75.

8 Internet World Stats. Interent usage statistics: the internet big picture. http://www. internetworldstats.com/stats.htm (accessed 15 Feb 2012)

9 Briones $\mathrm{R}$, Nan X, Madden $\mathrm{K}$, et al. When vaccines go viral: an analysis of HPV vaccine coverage on YouTube. Health Commun 2012;27:478-85.

$10 \mathrm{Yoo} \mathrm{JH}$, Kim J. Obesity in the new media: a content analysis of obesity videos on YouTube. Health Commun 2011;27:86-97.

11 Oh H, Rizo C, Enkin M, et al. What is eHealth: a systematic review of published definitions. J Med Internet Res 2005:7:e1.

12 Ahmed $\mathrm{OH}$, Sullivan SJ, Schneiders AG, et al. Concussion information online: evaluation of information quality, content and readability of concussion-related websites. Br J Sports Med 2012;46:675-83.

13 Bernstam EV, Walji MF, Sagaram S, et al. Commonly cited website quality criteria are not effective at identifying inaccurate online information about breast cancer. Cancer 2008;112:1206-13

14 Van Der Marel S, Duijvestein $\mathrm{M}$, et al. Quality of web-based information on inflammatory bowel diseases. Inflamm Bowel Dis 2009;15:1891-96.

15 Randeree E. Exploring technology impacts of healthcare 2.0 initiatives. Telemed J E Health 2009;15:255-60.

16 O'Reilly T. What is web 2.0: design patterns and business models for the next generation of software. Comm Strat 2007;65:17-37.

17 Eysenbach G. Medicine 2.0: social networking, collaboration, participation, apomediation, and openness. J Med Internet Res 2008;10:e22.

18 Sullivan SJ, Schneiders AG, Cheang CW, et al. 'What's happening?' A content analysis of concussion-related traffic on Twitter. Br J Sports Med 2012;46:258-63.

19 Ahmed OH, Sullivan SJ, Schneiders AG, et al. iSupport: do social networking sites have a role to play in concussion awareness? Disabil Rehabil 2010;32:1877-83.

20 Alexa: the web information company. Top sites: the top 500 sites on the web. http://www.alexa.com/ (accessed 25 May 2012).

21 YouTube. Press Room: Statistics. http://www.youtube.com/t/press_statistics (accessed 25 May 2012).

22 Grossman L. The beast with a billion eyes. Time 2012;179:38-43.

23 Cheng $X$, Dale C, Liu J. Statistics and social network of YouTube videos. 16th International Workshop on Quality of Service, IWQoS; 2008, Enschede.

24 Ache KA, Wallace LS. Human papillomavirus vaccination coverage on YouTube. Am J Prev Med 2008;35:389-92.

25 Richardson CG, Vettese L, Sussman S, et al. An investigation of smoking cessation video content on YouTube. Subst Use Misuse 2011:46:893-7.

26 Google. Google Adwords. https://adwords.google.com (accessed 8 Dec 2011).

27 Landis JR, Koch GG. The measurement of observer agreement for categorical data. Biometrics 1977;33:159-74.

28 Perotti V, Hair N. User experience in online social networks: a qualitative analysis of key activities and associated features. 44th Hawaii International Conference on System Sciences (HICSS); 2011, Honolulu.

29 Pew Internet \& American Life Project. Understanding the participatory news consumer. http://www.pewinternet.org/Reports/2010/Online-News.aspx?r=1 (accessed 20 Jan 2012).

30 Hughes S, Cohen D. Can online consumers contribute to drug knowledge? A mixed-methods comparison of consumer-generated and professionally controlled psychotropic medication information on the internet. J Med Internet Res 2011;13: e53.

31 YouTube. What should I put in my video description? http://support.google.com/ youtube/bin/answer.py?hl=en\&answer=57927 (accessed 20 Jan 2012)

32 YouTube. What's the difference between keywords and tags? http://support.google. com/youtube/bin/answer.py?hl=en\&answer=112948 (accessed 15 Jan 2012).

33 YouTube. How do I optimize my Video Watch Pages? http://support.google.com/ youtube/bin/answer.py?hl=en\&answer=141804 (accessed 20 Jan 2012).

34 McCrory PR, Berkovic SF. Video analysis of acute motor and convulsive manifestations in sport-related concussion. Neurology 2000;54:1488-91.

35 McIntosh AS, McCrory P, Comerford J. The dynamics of concussive head impacts in rugby and Australian rules football. Med Sci Sports Exerc 2001;32:1980-4.

36 Evans MF. '23 and $1 / 2 h^{\prime}$ goes viral: Top 10 learnings about making a health message that people give to one another. Br J Sports Med 2012;46:461-2. 